\title{
Study on Band Structure and the Mechanism of Two-dimensional Phononic Crystal with Boundary Rigid Constraints
}

\author{
Zhao Jingbo* \\ Science College \\ Air Force Engineering University \\ $\mathrm{Xi}$ an, China \\ E-mail: chjzjb@163.com \\ * Corresponding Author
}

Du Jun

Institute of Aeronautics and Astronautics

Air Force Engineering University

Xi'an, China

\author{
Yao Hong \\ Science College \\ Air Force Engineering University \\ $\mathrm{Xi}$ an, China
}

\author{
Zhao Xue \\ Science College \\ Air Force Engineering University \\ $\mathrm{Xi}$ an, China
}

\begin{abstract}
Paper studies the band structure changing with the matrix density and elastic modulus of the twodimensional phononic crystal in a square and circular rigid constraint boundary conditions, and the spring quality equivalent model theory is proposed. The research results show that the boundary constraint phononic crystal, the change of the band structure is the result of joint action both inside and outside boundary equivalent mass spring model. Boundary constraint phononic crystal band structure parameters and the change rule of research, provide a theoretical basis for a particular cutoff frequency phonon crystal design principles, which has guiding significance for the engineering control of low-frequency vibration noise.
\end{abstract}

Keywords-Phononic Crystal; Cut-off Frequency; Rigid Constraint; Noise; Low-Frequency

\section{INTRODUCTION}

In 1992, M. M. Sigalas and E. N. Economou theoretically for the first time confirmed that elastic wave band gaps in three-dimensional periodic lattice structure of the spherical scatter into the substrate material [1]. In 1993, M. S. Kushwaha and others put forward the concept of phononic crystal for the first time in the study of nickel/aluminum composite two-dimensional solid cycle [2], 2000, wuhan university, professor Liu Zhengyou proposed the local resonance type phononic crystal, the band gap of phononic crystal of the wave in the long run is greater than the lattice constant, breakthrough the limitation of the Bragg scattering mechanism, which created a new situation for the low-frequency vibration noise reduction and application of sonic crystals [3].

Ten years after the appearance of the local resonance mechanism, many important results have achieved around the phononic crystal of low frequency band gap. According to the band gap formation mechanism, it is mainly divided into several ways, one is introducing the harmonic oscillator in the unit cell, using the local resonance mechanism on low frequency band gaps [4-10], and local resonance band gap is usually associated with a single negative effective parameters, which is also the main mechanism of low frequency band gap. phononic crystal with negative effective parameters of, also known as generalized phonon crystal or metamaterials, was not a unified definition at the international. The second way is to use curly structure phononic crystal space method produce low-frequency band gap [11, 12], and the third way is to use rigid constraint phononic crystal, by using the methods of rigid constraint boundary or rigid inclusion induce the negative equivalent mass density and the presence of the cut-off frequency, resulting in a lower than the cutoff frequency of wide frequency band gap [13-15]. Low frequency vibration and noise control are always the difficult problems of engineering. Based on this analysis, the paper studied the different boundary rigid constraint conditions, the cut-off frequency with the equivalent mass of matrix and the change rule of equivalent modulus of elasticity. The quality of the spring equivalent model has carried on the quantitative description.

This paper studies the band gap characteristics of phononic crystal with different rigid constraint boundary. It is important for large aircraft cabin noise suppression, high distinguishability satellite vibration control, precision instrument vibration control and building earthquake protection. 


\section{SIMULATION MODEL OF CONSTRAINT PHONONIC CRYSTAL}

\section{A. Simulation Model of Circular Boundary Phononic Crystal}

Wherever Times is specified, Times Roman or Times New Roman may be used. If neither is available on your word processor, please use the font closest in appearance to Times. Avoid using bit-mapped fonts if possible. TrueType 1 or Open Type fonts are preferred. Please embed symbol fonts, as well, for math, etc. The unit and contracted brillouin zone map of two-dimensional phononic crystal can be seen in Figure 1. Tetragonal lattice constant $\mathrm{a}=0.1 \mathrm{~m}$, the circumference of boundary constraints radius $\mathrm{r}=\mathrm{a} / 10,8, \mathrm{a} / \mathrm{a} / 4,0.04 \mathrm{~m}, 0.045 \mathrm{~m}$, $0.047 \mathrm{~m}$ respectively. According to the former study of point and line constraints phononic crystals changing with the material, epoxy resin matrix in restricting the cutoff frequency is higher in phononic crystal, so take epoxy resin as matrix, the circular boundary constraints on the influence of the phonon crystal band structure. Matrix material for epoxy resin $(\mathrm{E}=4.35 \mathrm{GPa}$, rho $=1180 \mathrm{~kg} / \mathrm{m} 3$, $\mathrm{mu}=0.368)$, the material is set to lead $(\mathrm{E}=40.8 \mathrm{GPa}$, rho $=11600 \mathrm{~kg} / \mathrm{m} 3, \mathrm{mu}=0.369)$, using COMSOL software simulation, the simulation results as shown in figure.

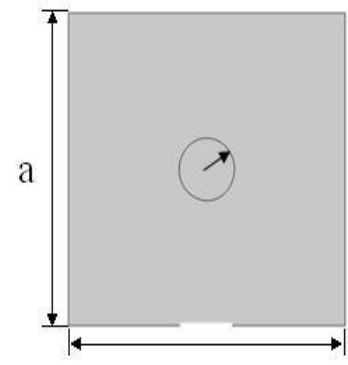

a

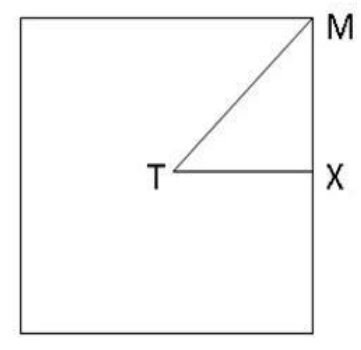

(a) Cell Unit

(b) Contracted Brillouin zone

Figure 1. Round Boundary Constraints of Two-Dimensional Phononic

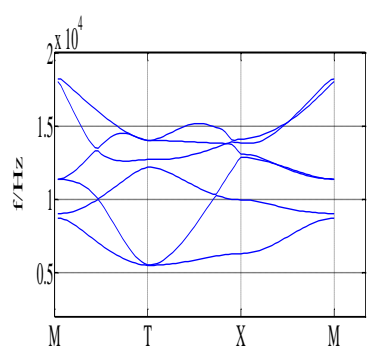
Crystal
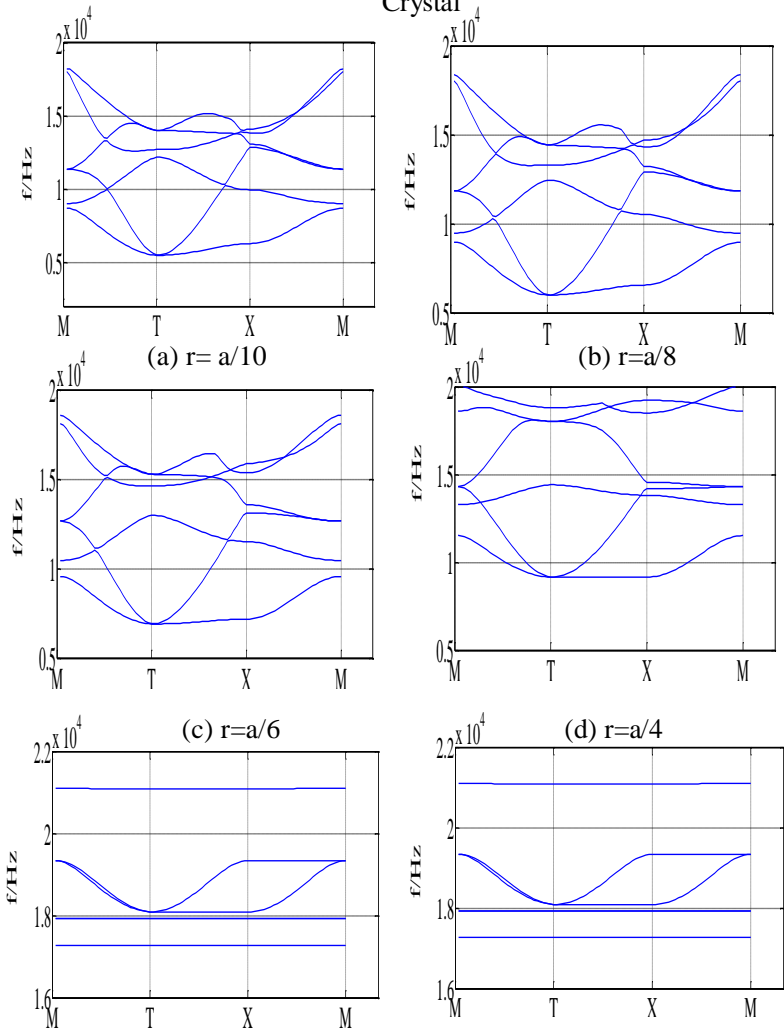

(e) $\mathrm{r}=0.04 \mathrm{~m}$

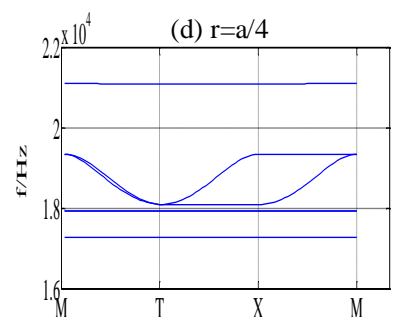

(f) $\mathrm{r}=0.045 \mathrm{~m}$

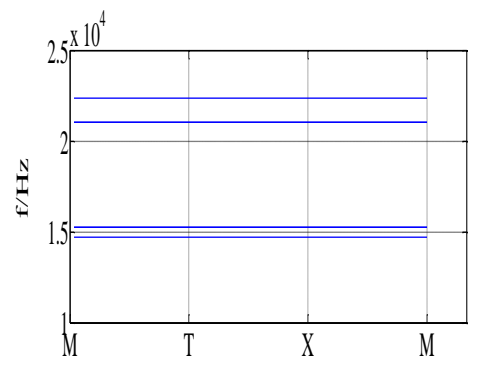

(g) $\mathrm{r}=0.047$

Figure 2. Band Structure with Different Radius of Phononic Crystal

Figure 2 is bandgap structure of boundary constraint phononic crystals with different radius. It can be seen the radius of the constraint has important effects on cut-off frequency. The cut-off frequency increases with the increase of constraint radius. When the radius is $0.047 \mathrm{~m}$, characteristic value curve into a straight line parallel to the direction of the arrow wavelengths, and vibration local state occurs according to the modal analysis, no displacement in the $\mathrm{x}$ axis and $\mathrm{y}$ axis. Figure 3 is the change rule of cutoff frequency with the boundary constraint circle radius changing. It can be seen from the graph, with the increase of boundary constraints circle radius, the cut-off frequency increases, and when the circular radius increases to $0.04 \mathrm{~m}$, cutoff frequency mutations, frequency increase rapidly at this point, the vibration modal is mainly for the local area. When the boundary circle radius continues to increase, cutoff frequency does not change, basic vibration modal all into local area. The results show that the radius of circular boundary constraint has important effects on phononic crystals bandgap structure, with the increase of circle radius, the cut-off frequency continues to increase, when the increases to a certain value, the cut-off frequency suddenly increases rapidly, and stable around a fixed frequency.

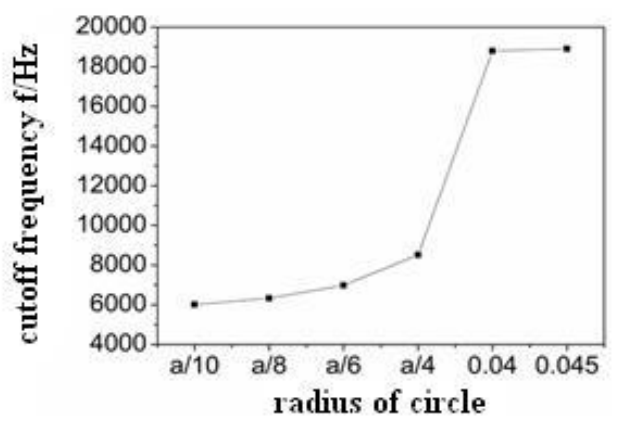

Figure 3. Cutoff Frequency Dependence on Boundary Constraint Circle Radius

\section{B. Simulation Model of Square Boundary Phononic Crystal}

The unit and contracted brillouin zone map of twodimensional square boundary constrain phononic crystal can be seen in Figure 4. Tetragonal lattice constant $\mathrm{a}=0.1$ $\mathrm{m}$, side $\mathrm{b}=0.05,0.07,0.09,0.1$, within the boundary material is set to lead $(\mathrm{E}=40.8 \mathrm{GPa}$, rho $=11600 \mathrm{~kg} / \mathrm{m} 3$, $\mathrm{mu}=0.369)$, the substrate material is still the epoxy resin $(\mathrm{E}=4.35 \mathrm{GPa}$, rho $=1180 \mathrm{~kg} / \mathrm{m} 3, \mathrm{mu}=0.368)$, using COMSOL software simulation. 

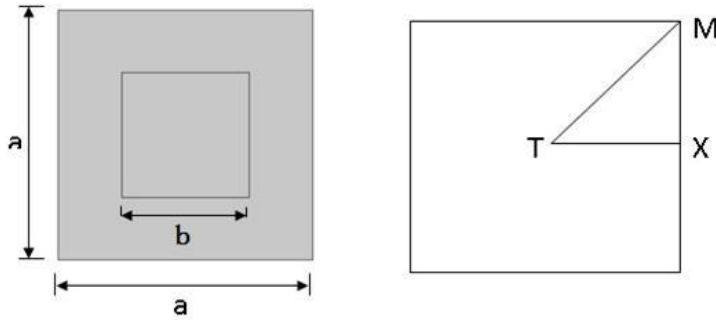

(a) Cell Unit (b) Contracted Brillouin Zone

Figure 4. Two-Dimensional Phononic Crystal with Square Boundary Constraints
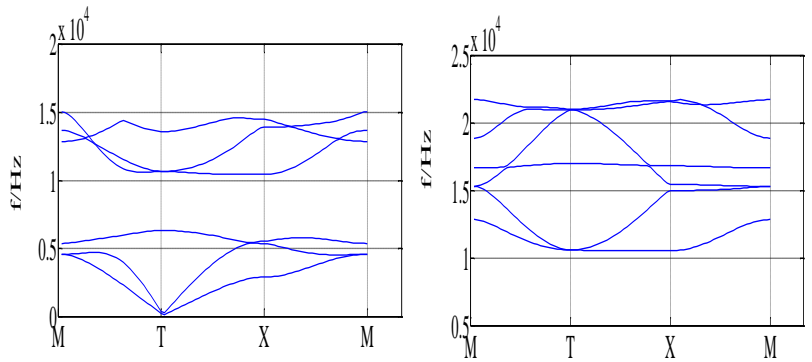

(a) $b=0.05 \mathrm{~m}$ no constrain

(b) $b=0.05 \mathrm{~m}$ square boundary constrain

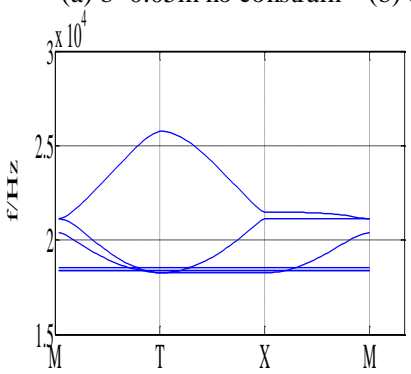

(c) $\mathrm{b}=0.07 \mathrm{~m}$

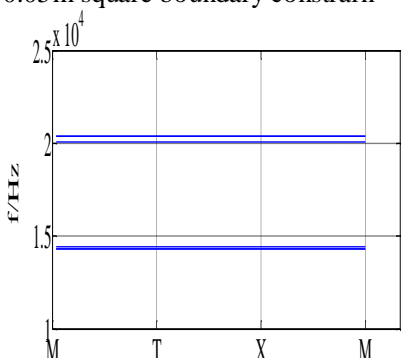

(d) $b=0.09 \mathrm{~m}$
Figure 5. Phononic Crystal Band Structure with Different Side Length of Square Boundary Constraint

Figure 5 (a) is phononic crystal without constraint with the side length of $0.05 \mathrm{~m}$, it can be seen from the diagram, the first frequency band gap range is $6010 \mathrm{~Hz}-6010 \mathrm{~Hz}$, and it can be found that the band gap of the mechanism is mainly for the Bragg scattering mechanism by the corresponding wavelength and lattice constant; Figure 5 (b, d) is phononic crystals band gap structure with different side length of square boundary constraint. It can be seen from the diagram, with the rising of the square side length, phonon crystal cut-off frequency increases, and when the side length of $0.05 \mathrm{~m}$, the cut-off frequency of $11500 \mathrm{~Hz}$; When the side length of $0.07 \mathrm{~m}$, the cut-off frequency of $18500 \mathrm{~Hz}$; When the side length of $0.09 \mathrm{~m}$, phonon crystal vibration modal for the local state, the cut-off frequency increases rapidly.

\section{EQUIVALENT MASS SPRING MODEL}

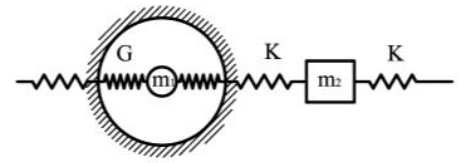

Figure 6. The Unit Equivalent Mass Spring Model with Circular Boundary Constraint at One Dimensional Direction
Figure 6 is one dimensional round boundary constraint direction unit equivalent mass spring model. Within the boundaries of constrain, the equivalent to quality $\mathrm{m} 1$ and spring stiffness coefficient G. Outside the boundary, matrix equivalent mass $\mathrm{m} 2$ and spring stiffness coefficient $\mathrm{K}$. It can be found by theory analysis, the equivalent model is consistent for matrix outside the circle boundary and point (line) constraint matrix, and cutoff frequency is inversely proportional to the RMS of matrix of the equivalent mass. From this perspective, along with the rising of the circle radius, matrix equivalent mass outside the circle boundary is reduced, cutoff frequency increases, that is consistent with the results of the simulation; Within the round boundaries, matrix equivalent mass $\mathrm{m} 1$ and $\mathrm{G}$ for spring stiffness coefficient, according to theoretical analysis in the topology, the matrix considered separately within the boundary, can be equivalent to a similar point (line) constraint matrix of the equivalent model on the one dimensional direction. The circular boundaries within the matrix cut-off frequency is inversely proportional to the equivalent mass matrix of RMS, is proportional to the spring stiffness coefficient. Thus, with the increase of circle boundary radius, matrix quality increase, the corresponding cutoff frequency should be reduced, and the simulation results (figure 3 ) shows that with the increase of radius of circular boundary, cutoff frequency on the rise.

From the above circle boundary constraint theory model analysis, in the circular boundary constraint phononic crystal, along with the rising of the circular boundary radius, both inside and outside boundary matrix can be equivalent to mass spring model. According to the comparison of simulation results and the theoretical model, it can be found that with the increase of radius of circular boundary, the equivalent mass spring model is dominant outside the boundary matrix, and the change of the cut-off frequency and boundary matrix of equivalent mass spring model is consistent with trends. When radius increases to a certain degree, cutoff frequency no longer change according to the modal analysis. It can be found that matrix within the boundary of dominant is dominant, and the local area state occurs within the local area, so the cutoff frequency is no longer improve with the increase of the radius.

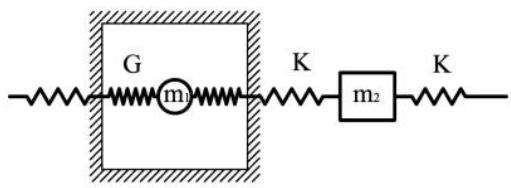

Figure 7. The Equivalent Mass Spring Model with Square Boundary Constraint

Figure 7 is unit equivalent mass spring model of square boundary constraint at one dimensional direction. As like circular boundary, within the boundaries of constrain, the equivalent to quality $\mathrm{m} 1$ and spring stiffness coefficient $\mathrm{G}$. Outside the boundary, matrix equivalent mass $\mathrm{m} 2$ and spring stiffness coefficient $\mathrm{K}$. It can be found in theory analysis, matrix and outside the boundary point (line) constraint matrix of the equivalent model is consistent, cutoff frequency is inversely proportional to the RMS of matrix of the equivalent mass, from this perspective, with 
the expanding of the square constraint boundary, the equivalent mass boundary in unit lattice matrix is reduced, so the cut-off frequency increases, which is consistent with the simulation results; Within the boundary of matrix equivalent mass block for $\mathrm{m} 1$ and stiffness coefficient for $\mathrm{G}$ spring, according to the formula, as square constraint boundary length increases, boundary matrix quality to increase, leading to lower cut-off frequency.

Overall, with the increase of square boundary length, the matrix outside the border first is dominant model, the cut-off frequency increases. When the boundary length increases to 0.09 above, the matrix within constraint boundary is dominant. According to the effective vibration mode, It can be found that matrix within the boundary keep local state, and the cutoff frequency will no longer increase.

\section{CONCLUSIONS}

Boundary constraint has significant effect on the band structure of phononic crystal, and band structure depends on the outcome of combined action of inside and outside boundary equivalent mass spring model. With the border size increases, the equivalent mass outside boundary model first is dominant, as the equivalent mass decrease gradually, the cut-off frequency according to the formula can be launched cut-off frequency increased; Size increases to a certain extent, when boundary model of equivalent mass inside is dominant, and a local state occurs, the cut-off frequency no longer improve; Also show that the boundary constraint phononic crystal, it is possible to realize the control of the phonon crystal band structure by changing parameters of substrate material, constraint type and constraint conditions. Due to special band structure rigidity, the research of the boundary constraint phononic crystal has important theoretical guiding significance for the engineering of low frequency vibration and noise control.

\section{ACKNOWLEDGMENT}

This work was supported by the National Natural Science Foundation of China (Grant No.11447147, 11504429), the National Science Foundation of Shaanxi Province, China (Grant No.2015JQ5155)

\section{REFERENCES}

[1] Sigalas M. M. and Ecomomou E.N.1992 J. Sound Vib. 158(2) 377382

[2] Sigalas M. M. and Ecomomou E.N. 1993 Solid State Commun. 86(3) $141-143$

[3] Liu Z. Y., Zhang X. X., Mao Y. W. 2000 Science 289 1734-1736

[4] Fang N, Xi D. J., Xu J. Y., Ambati M., Srituravanich W., Sun C. and Zhang X. 2006 Nat. Mater. 5 452-456

[5] Jing L., Wu J. H., Guan D. and Gao N.S.2014 J. Appl. Phys. 116 103514

[6] Li J. B., Wang Y. S., Zhang C. Z. 2013 J. Vib. and Acoust. 135 031015

[7] Celli P. and Gonella S. 2014 J. Appl. Phys. 115103502

[8] Ma J.H., Hou Z.L., and Badreddine M. Assouar. 2014 J. Appl. Phys. 115093508

[9] Liang Z. X. and Li J. S. 2012 Phys. Rev. Lett. 108114301

[10] Li Y., Liang B., Gu Z.M., Zou X. Y. and Cheng J. C. 2013 Sci. Rep. 32546

[11] Lee S. H., Park C. M., Seo Y.M., Wang Z. G. and Kim C.K.2009 Phys. Lett. A 373 4464-4469

[12] Yao S. S., Zhou X. M. and Hu G.K. 2010 New Journal of Physics 12103025

[13] Peng P., Qiu C.Y., Liu Z.Y., Wu Y. 2014 EPL 10646003

[14] Yao S. S., Zhou X. M. and Hu G.K. 2010 New Journal of Physics 12103025

[15] Peng P., Qiu C.Y., Liu Z.Y., Wu Y. 2014 EPL 10646003 